\title{
Fabrication and Characterization of ZnS/Diamond-Like Carbon Core-Shell Nanowires
}

\author{
Jung Han Kim, ${ }^{1,2}$ Seul Cham Kim, ${ }^{1}$ Do Hyun Kim, ${ }^{1}$ Kyu Hwan Oh, \\ Woong-Ki Hong, ${ }^{2}$ Tae-Sung Bae, ${ }^{2}$ and Hee-Suk Chung ${ }^{2}$ \\ ${ }^{1}$ Department of Materials Science and Engineering, Seoul National University, Seoul 151-742, Republic of Korea \\ ${ }^{2}$ Jeonju Center, Korea Basic Science Institute, Jeonju, Jeollabuk-do 561-180, Republic of Korea
}

Correspondence should be addressed to Tae-Sung Bae; chemipia@kbsi.re.kr and Hee-Suk Chung; hschung13@kbsi.re.kr

Received 4 November 2015; Revised 5 February 2016; Accepted 8 February 2016

Academic Editor: Meiyong Liao

Copyright (C) 2016 Jung Han Kim et al. This is an open access article distributed under the Creative Commons Attribution License, which permits unrestricted use, distribution, and reproduction in any medium, provided the original work is properly cited.

\begin{abstract}
We fabricated ZnS/diamond-like carbon (DLC) core-shell heterostructure nanowire using a simple two-step process: the vaporliquid-solid method combined with radio frequency plasma enhanced chemical vapor deposition (rf PECVD). As a core nanowire, $\mathrm{ZnS}$ nanowires with face-centered cubic structure were synthesized with a sputtered Au thin film, which exhibit a length and a diameter of $\sim 10 \mu \mathrm{m}$ and $\sim 30-120 \mathrm{~nm}$. After rf PECVD for DLC coating, The length and width of the dense ZnS/DLC core-shell nanowires were a range of $\sim 10 \mu \mathrm{m}$ and $50-150 \mathrm{~nm}$, respectively. In addition, ZnS/DLC core-shell nanowires were characterized with scanning transmission electron microscopy. From the results, the products have flat and uniform DLC coating layer on ZnS nanowire in spite of high residual stress induced by the high $\mathrm{sp}^{3}$ fraction. To further understanding of the DLC coating layer, Raman spectroscopy was employed with ZnS/DLC core-shell nanowires, which reveals two Raman bands at $1550 \mathrm{~cm}^{-1}(\mathrm{G}$ peak) and $1330 \mathrm{~cm}^{-1}$ (D peak). Finally, we investigated the optical properties from ultraviolet to infrared wavelength region using ultravioletvisible (UV-Vis) and Fourier transform infrared (FT-IR) spectrometry. Related to optical properties, ZnS/DLC core-shell nanowires exhibit relatively lower absorbance and higher IR transmittance than that of $\mathrm{ZnS}$ nanowires.
\end{abstract}

\section{Introduction}

Since Wagner and Ellis first discovered Si whiskers in 1964, one-dimensional (1D) nanostructures such as nanowires, nanobelts, and nanotubes have been under active development owing to their novel physical and chemical properties associated with their unique size and dimensionality [1-5]. The nanostructures have been mainly utilized as interconnections in a variety of nanoscale electronic/optical devices, single-electron transistors, light-emitting diodes, gas/chemical sensors, and photodetectors [6-10]. Among many semiconductor materials, $\mathrm{ZnS}$ is an important II-VI group semiconductor with fascinating excellent optical properties (direct bandgap energy $=3.68 \mathrm{eV}$ at $300 \mathrm{~K}$ ) and thus has been recently considered as the most promising material for phosphors in cathode-ray tubes and flat-panel displays, ultraviolet-light-emitting diodes, and injection lasers [1114]. Extensive efforts have been made on the synthesis of
1D $\mathrm{ZnS}$ nanowires, nanotubes, nanobelts, and their complex assemblies [15-18].

The stability of nanowires is a key factor that corresponds to their practical applications used on multiassembled nanoscale electrodevices $[19,20]$. In order to fully exploit $\mathrm{ZnS}$ nanowires as nanodevices, waveguides, and so forth, it is very important to passivate the surface of the $\mathrm{ZnS}$ nanowires, which have structures that exhibit high-chemical reactivity with respect to the deformation, oxidation, corrosion, and contamination of these structures. In this regard, several groups have investigated the protection of $\mathrm{ZnS}$ nanowires with boron nitride $(\mathrm{BN})$ or silicon dioxide $\left(\mathrm{SiO}_{2}\right)[21,22]$. However, core-shell heterostructured $\mathrm{ZnS}$ nanowires used in various encapsulating nanomaterials are not yet understood in detail.

In this study, we aimed to introduce $\mathrm{ZnS}$ and diamondlike carbon (DLC) core-shell heterostructure nanowires to gain better insight into their applications towards nanoscale 
electrodevices. DLC is well-known as $\mathrm{sp}^{2}$ and $\mathrm{sp}^{3}$ hybridized amorphous carbon materials and can be used in various applications due to the superior mechanical properties with a low friction coefficient, wear resistance, chemical inertness, and biocompatibility [23-25]. We fabricated ZnS/DLC coreshell heterostructure nanowires by a simple two-step process: the vapor-liquid-solid (VLS) method combined with radio frequency plasma enhanced chemical vapor deposition ( $\mathrm{rf}$ PECVD). This fabrication is expected to open new possibilities for realizing of a lot of semiconductor nanostructures with DLC coating.

\section{Experimental Methods}

A core nanowire, $\mathrm{ZnS}$ nanowire, was synthesized by a metalorganic chemical vapor deposition (MOCVD) process using a $\mathrm{Zn}\left(\mathrm{S}_{2} \mathrm{CNEt}_{2}\right)_{2}$ single molecular precursor in a horizontal furnace (Nextron, LABSYS CGF-5000) with a $30 \mathrm{~mm}$ diameter quartz tube [26]. $\mathrm{Zn}\left(\mathrm{S}_{2} \mathrm{CNEt}_{2}\right)_{2}$ powder (Sigma Aldrich) was then placed upstream of a quartz tube and $\mathrm{Au}$ sputtered thin film (thickness $\sim 30 \mathrm{~nm}$ ) coated Si substrate was positioned downstream of the quartz tube that was initially evacuated till $\sim 5$ mTorr. Subsequently, Ar flow in the quartz tube was set to $100 \mathrm{sccm}$ (SCCM denotes cubic centimeter per minute at standard temperature and pressure (STP)), which enabled the quartz tube to achieve a constant pressure of 300 Torr. After the furnace was stabilized at the process pressure of 300 Torr, the precursor was slowly pushed into the high temperature zone of the furnace and the growth of the $\mathrm{ZnS}$ nanowires proceeded for 10 minutes. After the processing of the synthesis, the furnace was cooled down to room temperature.

The as-synthesized $\mathrm{ZnS}$ nanowires were moved to the $\mathrm{rf}$ PECVD system with $13.56 \mathrm{MHz}$. As for PECVD process, vacuum chamber was evacuated to $4 \times 10^{-5}$ Torr for ultraclean and stable plasma generation. Methane $\left(\mathrm{CH}_{4}\right)$ with a flow of $10 \mathrm{sccm}$ was then put into the chamber, which was allowed to reach $3 \times 10^{-2}$ Torr for the DLC deposition conditions. To facilitate the deposition of a thin DLC coating on the $\mathrm{ZnS}$ nanowires, rf power was applied at $100 \mathrm{~W}$ for 60 seconds. Different from the as-synthesized $\mathrm{ZnS}$ nanowires in gray, the $\mathrm{ZnS} / \mathrm{DLC}$ core-shell nanowires were a light-yellow color.

The obtained $\mathrm{ZnS} / \mathrm{DLC}$ core-shell nanowires were analyzed by X-ray diffraction (XRD, Bruker D8 Advance), scanning electron microscopy (SEM, FEI NOVA 200), transmission electron microscopy (TEM, JEOL-3000F), energy dispersive spectroscopy (EDS, Oxford INCA), Raman spectroscopy (LabRam HR), ultraviolet-visible spectroscopy (UV-Vis, Agilent Technologies Cary 5000), and Fourier transform infrared spectrometry (FT-IR, Bruker IFS-66/S).

\section{Results and Discussion}

Figure 1 shows a representative XRD pattern of ZnS/DLC core-shell nanowires. All the diffraction peaks were identified as a face-centered cubic (FCC) phase of ZnS (JCPDS: 050566), while XRD peaks of DLC were hardly detected due to its amorphous structure.

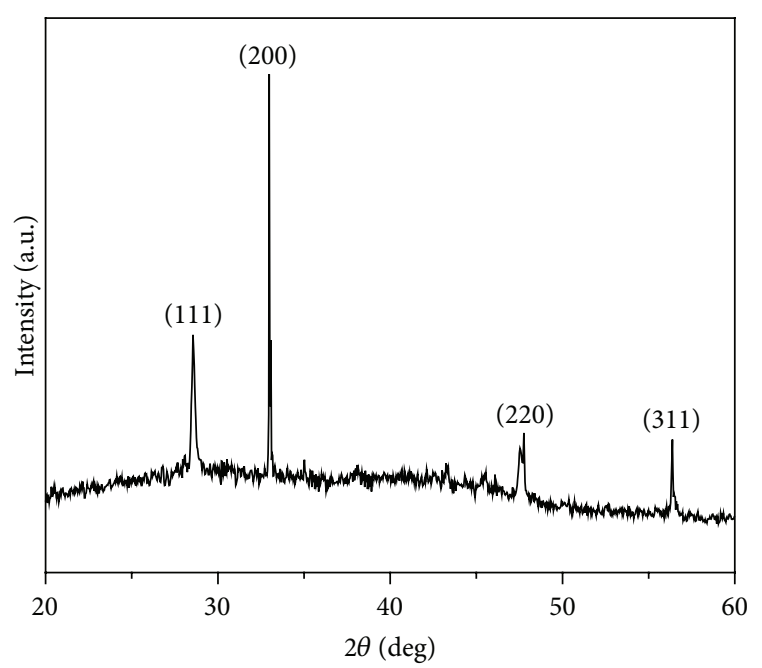

FIGURE 1: X-ray diffraction pattern of ZnS/DLC core-shell nanowires.

A morphological study of the ZnS/DLC core-shell nanowires was performed using SEM equipped with a dual beam focused ion beam system. Figure 2(a) displays a low-magnification SEM image of the ZnS/DLC core-shell nanowires. The length and width of the dense ZnS/DLC core-shell nanowires on $\mathrm{Si}$ substrate were in a range of $\sim 10 \mu \mathrm{m}$ and 50-150 nm, respectively. The inset of Figure 2(a) shows SEM image of the as-synthesized $\mathrm{ZnS}$ nanowires with a length and a width of $\sim 10 \mu \mathrm{m}$ and $\sim 30-120 \mathrm{~nm}$, respectively. Figure 2(b) shows a high magnification SEM image of ZnS/DLC coreshell nanowires. Using the transmission mode of SEM, the outer layer of the DLC on the $\mathrm{ZnS}$ core nanowires can be clearly seen to have a thickness of $\sim 20 \mathrm{~nm}$. The inset of Figure 2(b) shows an enlarged SEM image of the as-synthesized $\mathrm{ZnS}$ nanowires. As shown in the inset of Figure 2(b), Au catalyst is clearly observed at the tip of an as-synthesized $\mathrm{ZnS}$ nanowire, indicating that the growth of $\mathrm{ZnS}$ nanowires was caused by the VLS method.

A detailed structural and chemical analysis of ZnS/DLC core-shell nanowires was conducted by TEM. For the sample preparation of TEM analysis, ZnS/DLC nanowires were directly dispersed onto a $\mathrm{SiO}_{2}$ thin film on a TEM grid. Figure 3(a) shows a low-magnification TEM image of a ZnS/DLC nanowire, which indicated that the $\mathrm{ZnS} / \mathrm{DLC}$ nanowire is $\sim 80 \mathrm{~nm}$ in diameter with a flat interface between the $\mathrm{ZnS}$ and the DLC core-shell layer. A high resolution TEM (HRTEM) was employed for the detailed investigation of the structure of $\mathrm{ZnS} / \mathrm{DLC}$ core-shell nanowires. Figure 3(b) presents a HRTEM image of the ZnS/DLC core-shell nanowires and shows a clear interface between the surface of the assynthesized ZnS nanowire and the outer layer of DLC. As observed in the inner $\mathrm{ZnS}$ nanowire, it shows exactly (111) lattice plane with a d-spacing of $\sim 3.12 \AA$, which exhibits the $\mathrm{ZnS}$ nanowire formed along the [111] growth direction. This was confirmed by fast Fourier transformation (FFT), as shown in the inset of Figure 3(b), clearly indicating the single-

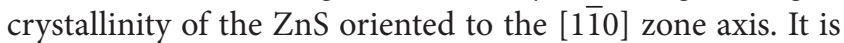
worth mentioning that the interface between the $\mathrm{ZnS}$ and 


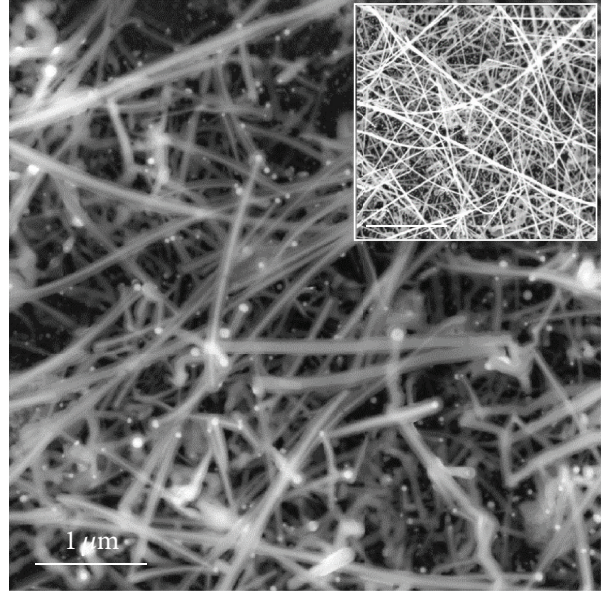

(a)

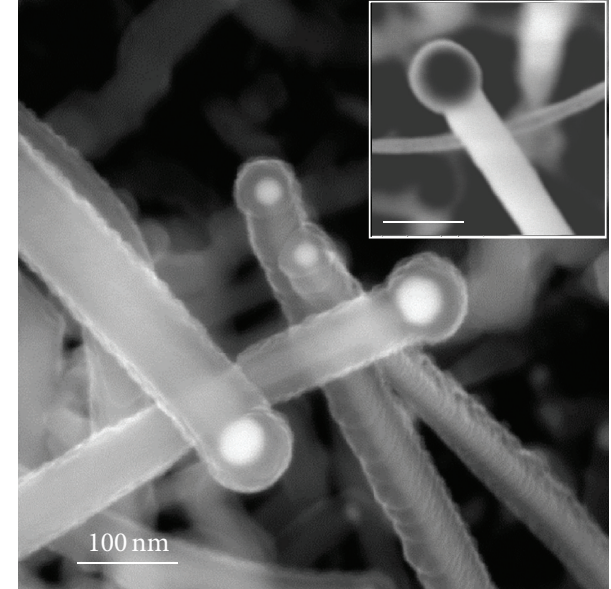

(b)

FIGURE 2: (a) Low-magnification SEM image of ZnS/DLC core-shell nanowires (inset: SEM image of as-synthesized ZnS nanowires. Scale bar is $1 \mu \mathrm{m}$.). (b) High-magnification SEM image of ZnS/DLC core-shell nanowires (inset: enlarged SEM image of as-synthesized ZnS nanowires. Scale bar is $100 \mathrm{~nm}$.).

the DLC layer is very flat and uniform without any pretreatment performed on the $\mathrm{ZnS}$ nanowires. DLC is generally known to be a material that is difficult to deposit on any substrate since it has high residual stress induced by the high $\mathrm{sp}^{3}$ fraction [27]. Therefore, pretreatments, for instance, ion bombardment on an arbitrary substrate, have been applied to the DLC deposition to promote good adhesion between the film and the substrate.

To further investigate the chemical information of the $\mathrm{ZnS} / \mathrm{DLC}$ core-shell nanowire, we conducted a scanning TEM (STEM) annular dark field (ADF) analysis with EDS line scan profile. Figure 3(c) presents ADF image and the EDS line-profile analysis crossed with the growth direction laid on the ADF image of the ZnS/DLC core-shell nanowire. This evidently shows that only zinc (red) and sulfur (blue) were present within the $\mathrm{ZnS}$ nanowire, which has an intrinsic chemical composition without interdiffusion at the interface between ZnS and DLC. Carbon (green), on the other hand, was uniformly distributed along the width direction of the core-shell nanowires. Figure 3(d) illustrates EDS spectrum obtained from the core-shell, which indicates that $\mathrm{Cu}, \mathrm{Si}$, and $O$ have originated from the TEM grid.

In order to gain further understanding of the DLC bonding state, Raman spectroscopy was employed. Figure 4(a) displays the Raman spectrum of the ZnS/DLC core-shell nanowires, which exhibits two kinds of Raman peaks that were observed at $1550 \mathrm{~cm}^{-1}$ ( $\mathrm{G}$ peak) due to $\mathrm{E}_{2 \mathrm{~g}}$ symmetry and vibration of $\mathrm{sp}^{2}$ bonding in graphite carbon, and at $1330 \mathrm{~cm}^{-1}$ (D peak) regarding the breathing modes of $\mathrm{sp}^{2}$ bonding in disordered graphitic carbon. Among many kinds of DLC materials, DLC deposited on ZnS nanowires are the most likely typical hydrogenated diamond-like amorphous carbon, which is in good agreement with the previous results of DLC deposited with PECVD [28].
To explore the optical properties of the ZnS/DLC coreshell nanowires, we conducted UV-Vis and FT-IR spectrometry from ultraviolet to infrared wavelength region. As for the measurement of the optical spectrum, we directly dispersed ZnS/DLC core-shell and ZnS nanowires onto a glass substrate and the measurements were made at least 20 times on each sample to ensure reliability. Firstly, we investigated absorption properties ZnS/DLC core-shell and $\mathrm{ZnS}$ nanowires between ultraviolet and infrared wavelength regime using UV-Vis spectrometry. As shown in Figure 4(b), absorbance of $\mathrm{ZnS} / \mathrm{DLC}$ nanowires is relatively lower than that of $\mathrm{ZnS}$ nanowires overall measured wavelength, which might be due to reflectance reduction from DLC coating (DLC refractive index: 1.8, ZnS refractive index 2.3). Further, we observed absorption peaks $313 \mathrm{~nm}$ and $326 \mathrm{~nm}$ of ZnS/DLC core-shell and ZnS nanowires, respectively. Absorption peak of bulk $\mathrm{ZnS}$ is generally known as $335 \mathrm{~nm}$. In this regard, UV-Vis analysis indicates blue shifted spectrum of 9 22 $\mathrm{nm}$ both $\mathrm{ZnS} / \mathrm{DLC}$ core-shell and $\mathrm{ZnS}$ nanowires, which is conceivably attributed to quantum confinement effect $[29,30]$. Subsequently, we performed FT-IR analysis in order to gain IR transmittance. The FT-IR spectrum at a wavenumber range of $1400 \mathrm{~cm}^{-1}$ to $4000 \mathrm{~cm}^{-1}$, as shown in Figure 4(c), reveals that the ZnS/DLC core-shell nanowires (black) of IR transmittance were enhanced with values of $\sim 1.2 \%$ higher than those of the $\mathrm{ZnS}$ nanowires. In general, $\mathrm{ZnS}$ and DLC combined structure is very well known for enhanced transmittance system in visible and infrared wavelength period such as solar cell, military device [31]. More generally, DLC provides lower refractive index surface $(\sim$ $1.8)$ to inner $\mathrm{ZnS}$ nanowire. However, in our $\mathrm{ZnS} / \mathrm{DLC}$ coreshell and $\mathrm{ZnS}$ nanowires, difference of IR transmittance is about $1.2 \%$. Even though IR transmittance value between $\mathrm{ZnS} / \mathrm{DLC}$ and $\mathrm{ZnS}$ nanowires seems to be small, we guess that this enhanced IR transmittance is due to DLC coating layer. 


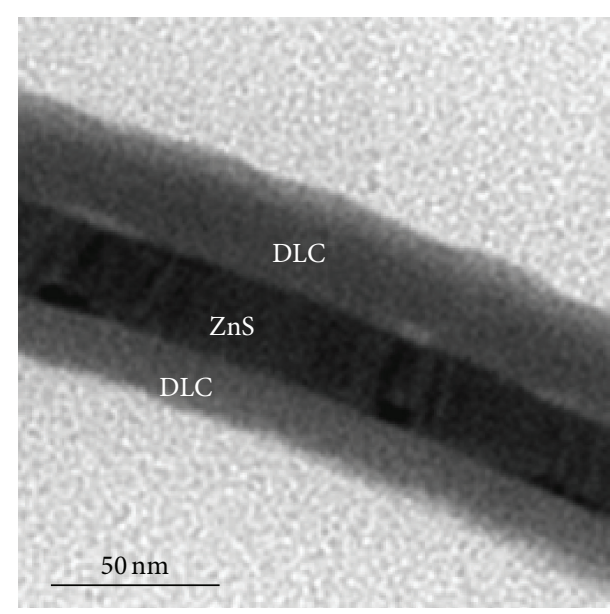

(a)

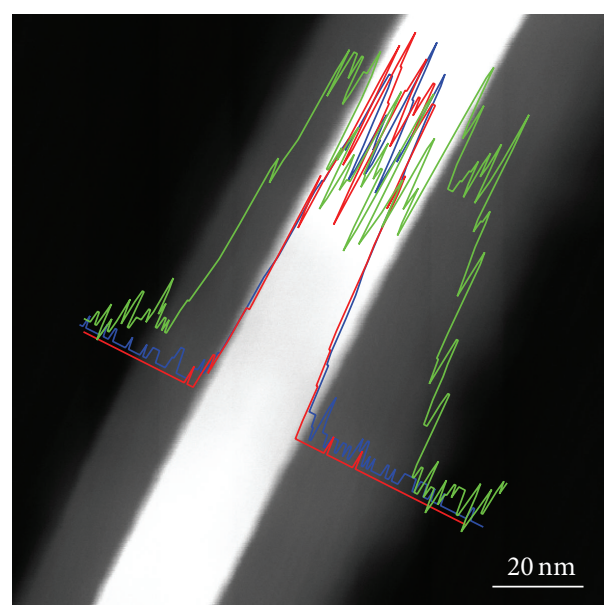

(c)

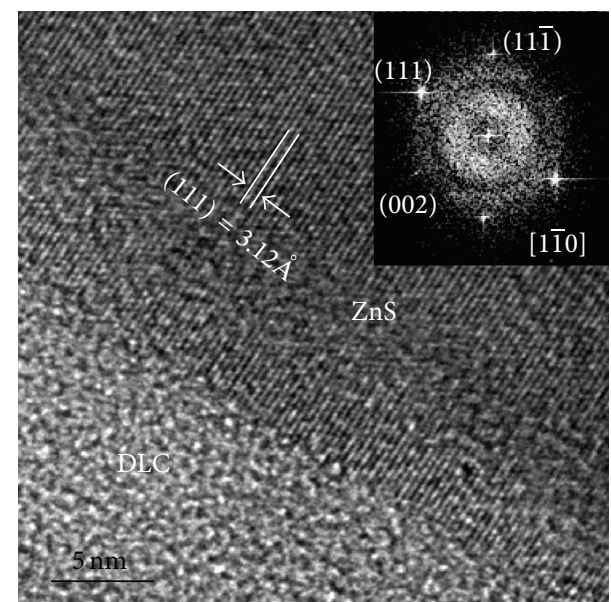

(b)

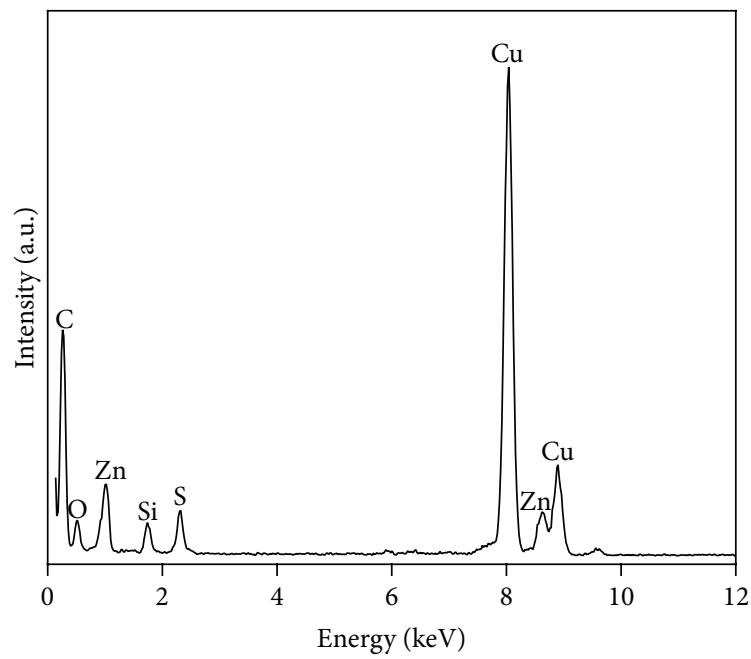

(d)

FIGURE 3: (a) The low-magnification TEM image of a ZnS/DLC nanowire. (b) HRTEM image of ZnS/DLC core-shell nanowires revealing growth along the [111] direction (inset: corresponding FFT pattern obtained by [1히] zone axis). (c) ADF-STEM image of ZnS/DLC coreshell nanowires with corresponding EDS line-scan profile along the width direction (red: Zn, blue: S, and green: C). (d) EDS spectrum of (c).

Further, we suggest that difference of IR transmittance is only due to single surface reflection of nanoscale compared to bulk $\mathrm{ZnS} / \mathrm{DLC}$ system including single and multiple reflection within visible and infrared region.

The fabrication process developed in this work is a very simple two-step process consisting of Au-catalyzed VLS growth and sequential rf PECVD and is able to synthesize $\mathrm{ZnS} / \mathrm{DLC}$. There is still a limitation to obtaining fully uniform core-shell heterostructured nanowires due to the randomly oriented nature of the initial nanowires on the substrate. However, our fabrication technique may open up the superior stack coverage on $1 \mathrm{D}$ nanostructures using DLC as an outer layer deposited by common PECVD. Moreover, this new process may be more feasible and effective platform for multiassembled nanodevices with elaborate deposition technology.

\section{Conclusions}

In summary, $\mathrm{ZnS} /$ diamond-like carbon core-shell heterostructure nanowires were successfully synthesized by VLS growth of $\mathrm{ZnS}$ nanowires and a sequential rf PECVD process. ZnS nanowires were initially synthesized with a width of 70$80 \mathrm{~nm}$ and DLC layers were continuously deposited on the surface of $\mathrm{ZnS}$ nanowires with a thickness of $20-30 \mathrm{~nm}$. The detailed analysis of the carbon structure of the DLC with Raman spectroscopy confirmed that two kinds of Raman bands observed at $1550 \mathrm{~cm}^{-1}$ (G peak) and at $1330 \mathrm{~cm}^{-1}$ (D peak) indicated DLC to be hydrogenated diamond-like amorphous carbon. Through UV-Vis spectrometry for optical properties investigation, absorbance of $\mathrm{ZnS} / \mathrm{DLC}$ core-shell nanowires seems relatively lower than that of $\mathrm{ZnS}$ nanowires with blue-shift of absorption peak within wavelength of $300 \sim 500 \mathrm{~nm}$. As for FT-IR spectrometry analysis between 


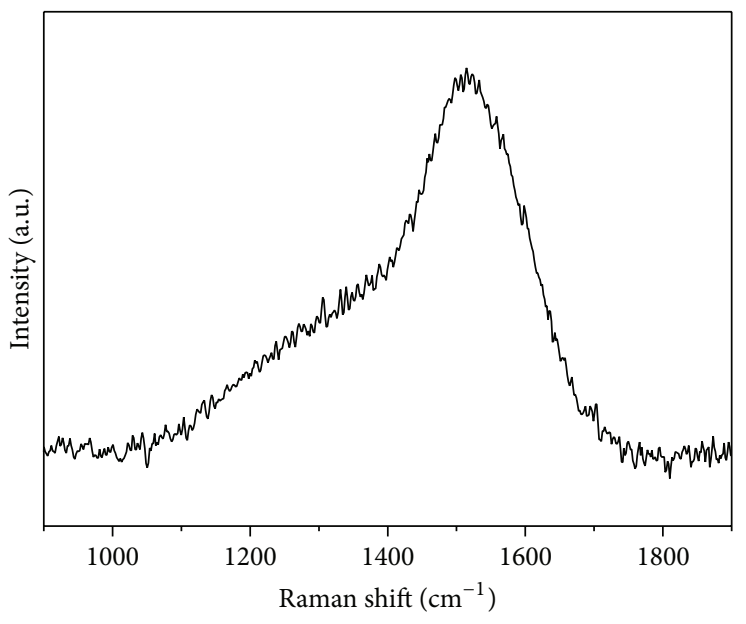

(a)

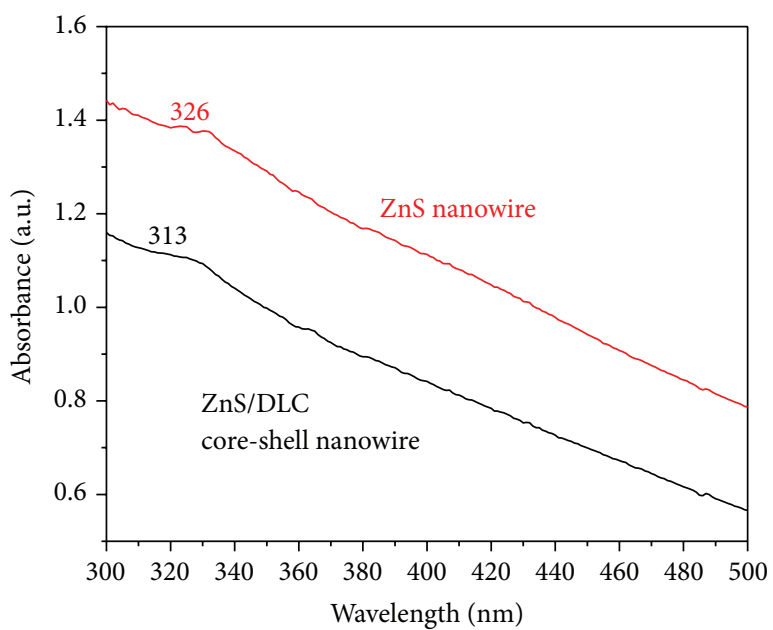

(b)

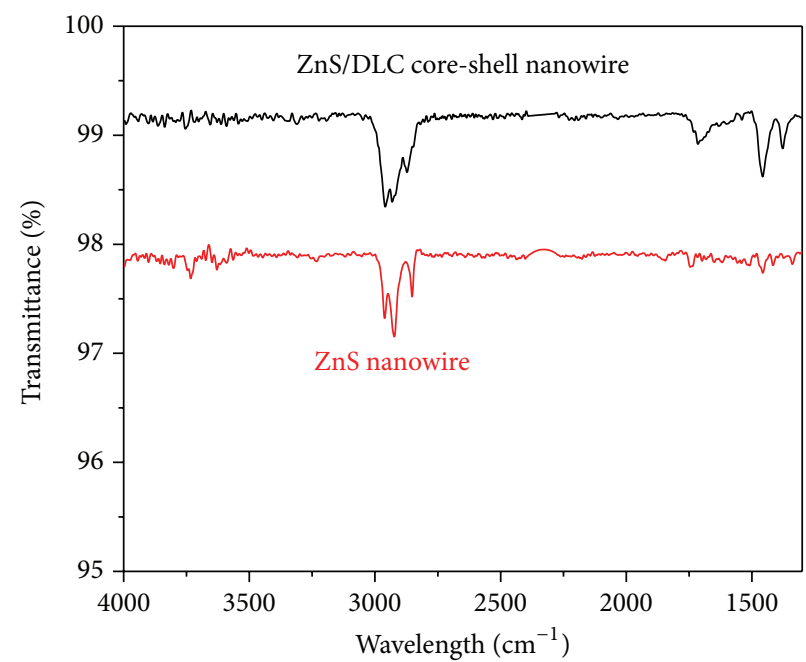

(c)

FIGURE 4: (a) Raman spectrum of ZnS/DLC core-shell nanowires. (b) UV-Vis absorption spectrum and (c) FT-IR spectrum of as-synthesized $\mathrm{ZnS}$ nanowire (red) and $\mathrm{ZnS} / \mathrm{DLC}$ (black) core-shell nanowire.

$1400 \mathrm{~cm}^{-1}$ and $4000 \mathrm{~cm}^{-1}$ wavelength at infrared regime, we observed enhanced IR transmittance of ZnS/DLC coreshell nanowires with a value of about $1.2 \%$ compared to $\mathrm{ZnS}$ nanowires. The heterostructure core-shell nanowire developed in the present work can be applied to a wide range of nanoscale electrodevices, which not only introduces novel perspectives for applying DLC on a semiconductor surface but also establishes a nanoscale fabrication technique for future nanodevices.

\section{Conflict of Interests}

The authors declare that there is no conflict of interests regarding the publication of this paper.

\section{Acknowledgments}

This work was supported by National Research Foundation of Korea (NRF) grant funded by the Korea government
(MSIP) (no. 2015R1C1A1A01052727) and Korea Basic Science Institute (KBSI) (KBSI, no. C35928).

\section{References}

[1] R. S. Wagner and W. C. Ellis, "Vapor-liquid-solid mechanism of single crystal growth," Applied Physics Letters, vol. 4, no. 5, pp. 89-90, 1964.

[2] N. P. Dasgupta, J. Sun, C. Liu et al., "25th Anniversary article: semiconductor nanowires-synthesis, characterization, and applications," Advanced Materials, vol. 26, no. 14, pp. 2137-2183, 2014.

[3] R. S. Devan, R. A. Patil, J.-H. Lin, and Y.-R. Ma, "Onedimensional metal-oxide nanostructures: recent developments in synthesis, characterization, and applications," Advanced Functional Materials, vol. 22, no. 16, pp. 3326-3370, 2012.

[4] Y. B. Guo, L. Xu, H. B. Liu, Y. J. Li, C. M. Che, and Y. L. $\mathrm{Li}$, "Self-assembly of functional molecules into 1D crystalline nanostructures," Advanced Materials, vol. 27, no. 6, pp. 985-1013, 2015. 
[5] D. Moore and Z. L. Wang, "Growth of anisotropic onedimensional ZnS nanostructures," Journal of Materials Chemistry, vol. 16, no. 40, pp. 3898-3905, 2006.

[6] D. Jariwala, V. K. Sangwan, L. J. Lauhon, T. J. Marks, and M. C. Hersam, "Carbon nanomaterials for electronics, optoelectronics, photovoltaics, and sensing," Chemical Society Reviews, vol. 42, no. 7, pp. 2824-2860, 2013.

[7] X. Guo, Y. B. Ying, and L. M. Tong, "Photonic nanowires: from subwavelength waveguides to optical sensors," Accounts of Chemical Research, vol. 47, no. 2, pp. 656-666, 2014.

[8] S. R. Suryawanshi, S. S. Warule, S. S. Patil, K. R. Patil, and M. A. More, "Vapor-liquid-solid growth of one-dimensional tin sulfide $(\mathrm{SnS})$ nanostructures with promising field emission behavior," ACS Applied Materials and Interfaces, vol. 6, no. 3, pp. 2018-2025, 2014.

[9] F. S. Kim, G. Ren, and S. A. Jenekhe, "One-dimensional nanostructures of $\pi$-conjugated molecular systems: assembly, properties, and applications from photovoltaics, sensors, and nanophotonics to nanoelectronics," Chemistry of Materials, vol. 23, no. 3, pp. 682-732, 2011.

[10] F.-X. Xiao, J. Miao, H. B. Tao et al., "One-dimensional hybrid nanostructures for heterogeneous photocatalysis and photoelectrocatalysis," Small, vol. 11, no. 18, pp. 2115-2131, 2015.

[11] M. Bredol and J. Merikhi, "ZnS precipitation: morphology control," Journal of Materials Science, vol. 33, no. 2, pp. 471-476, 1998.

[12] Y. F. Zhu, D. H. Fan, and W. Z. Shen, "A general chemical conversion route to synthesize various $\mathrm{ZnO}$-based core/shell structures," Journal of Physical Chemistry C, vol. 112, no. 28, pp. 10402-10406, 2008.

[13] X. S. Fang, Y. Bando, M. Y. Liao et al., "Single-crystalline ZnS nanobelts as ultraviolet-light sensors," Advanced Materials, vol. 21, no. 20, pp. 2034-2039, 2009.

[14] Y. Jiang, X.-M. Meng, J. Liu, Z.-R. Hong, C.-S. Lee, and S.-T. Lee, "ZnS nanowires with wurtzite polytype modulated structure," Advanced Materials, vol. 15, no. 14, pp. 1195-1198, 2003.

[15] Y.-C. Zhu, Y. Bando, D.-F. Xue, and D. Golberg, "Oriented assemblies of $\mathrm{ZnS}$ one-dimensional nanostructures," Advanced Materials, vol. 16, no. 9-10, pp. 831-834, 2004.

[16] X. S. Fang, L. Wu, and L. Hu, "ZnS nanostructure arrays: a developing material star," Advanced Materials, vol. 23, no. 5, pp. 585-598, 2011.

[17] X. S. Fang, T. Y. Zhai, U. K. Gautam et al., "ZnS nanostructures: from synthesis to applications," Progress in Materials Science, vol. 56, no. 2, pp. 175-287, 2011.

[18] X. S. Fang, Y. S. Bando, C. H. Ye, G. Z. Shen, and D. Golberg, "Shape- and size-controlled growth of ZnS nanostructures," Journal of Physical Chemistry C, vol. 111, no. 24, pp. 8469-8474, 2007.

[19] U. Khalilov, M. Yusupov, A. Bogaerts, and E. C. Neyts, "Selective plasma oxidation of ultrasmall Si nanowires," The Journal of Physical Chemistry C, vol. 120, no. 1, pp. 472-477, 2016.

[20] F. Cao, X. Xia, G. Pan, J. Chen, and Y. Zhang, "Construction of carbon nanoflakes shell on $\mathrm{CuO}$ nanowires core as enhanced core/shell arrays anode of lithium ion batteries," Electrochimica Acta, vol. 178, pp. 574-579, 2015.

[21] Y.-C. Zhu, Y. Bando, D.-F. Xue, F.-F. Xu, and D. Golberg, "Insulating tubular BN sheathing on semiconducting nanowires," Journal of the American Chemical Society, vol. 125, no. 47, pp. 14226-14227, 2003.
[22] X. Fan, X.-M. Meng, X.-H. Zhang, S.-K. Wu, and S.-T. Lee, "Formation of $\mathrm{ZnS} / \mathrm{SiO}_{2}$ nanocables," Applied Physics Letters, vol. 86, no. 17, Article ID 173111, pp. 1-3, 2005.

[23] J. Robertson, "Diamond-like amorphous carbon," Materials Science and Engineering: R: Reports, vol. 37, no. 4-6, pp. 129282, 2002.

[24] A. Grill, "Diamond-like carbon coatings as biocompatible materials-an overview," Diamond and Related Materials, vol. 12, no. 2, pp. 166-170, 2003.

[25] A. H. Lettington, "Applications of diamond-like carbon thin films," Carbon, vol. 36, no. 5-6, pp. 555-560, 1998.

[26] Z. Q. Wang, X. D. Liu, J. F. Gong, H. B. Huang, S. L. Gu, and S. G. Yang, "Epitaxial growth of $\mathrm{ZnO}$ nanowires on $\mathrm{ZnS}$ nanobelts by metal organic chemical vapor deposition," Crystal Growth and Design, vol. 8, no. 11, pp. 3911-3913, 2008.

[27] E. Liu, L. Li, B. Blanpain, and J. P. Celis, "Residual stresses of diamond and diamondlike carbon films," Journal of Applied Physics, vol. 98, no. 7, Article ID 073515, 2005.

[28] K. J. Clay, S. P. Speakman, N. A. Morrison, N. Tomozeiu, W. I. Milne, and A. Kapoor, "Material properties and tribological performance of rf-PECVD deposited DLC coatings," Diamond and Related Materials, vol. 7, no. 8, pp. 1100-1107, 1998.

[29] Y. C. Li, X. H. Li, C. H. Yang, and Y. F. Li, "Ligand-controlling synthesis and ordered assembly of $\mathrm{ZnS}$ nanorods and nanodots," The Journal of Physical Chemistry B, vol. 108, no. 41, pp. 16002-16011, 2004.

[30] A. Sengupta, B. Jiang, K. C. Mandal, and J. Z. Zhang, "Ultrafast electronic relaxation dynamics in $\mathrm{PbI} 2$ semiconductor colloidal nanoparticles: a femtosecond transient absorption study," The Journal of Physical Chemistry B, vol. 103, no. 16, pp. 3128-3137, 1999.

[31] A. Grill, "Diamond-like carbon: state of the art," Diamond and Related Materials, vol. 8, no. 2-5, pp. 428-434, 1999. 

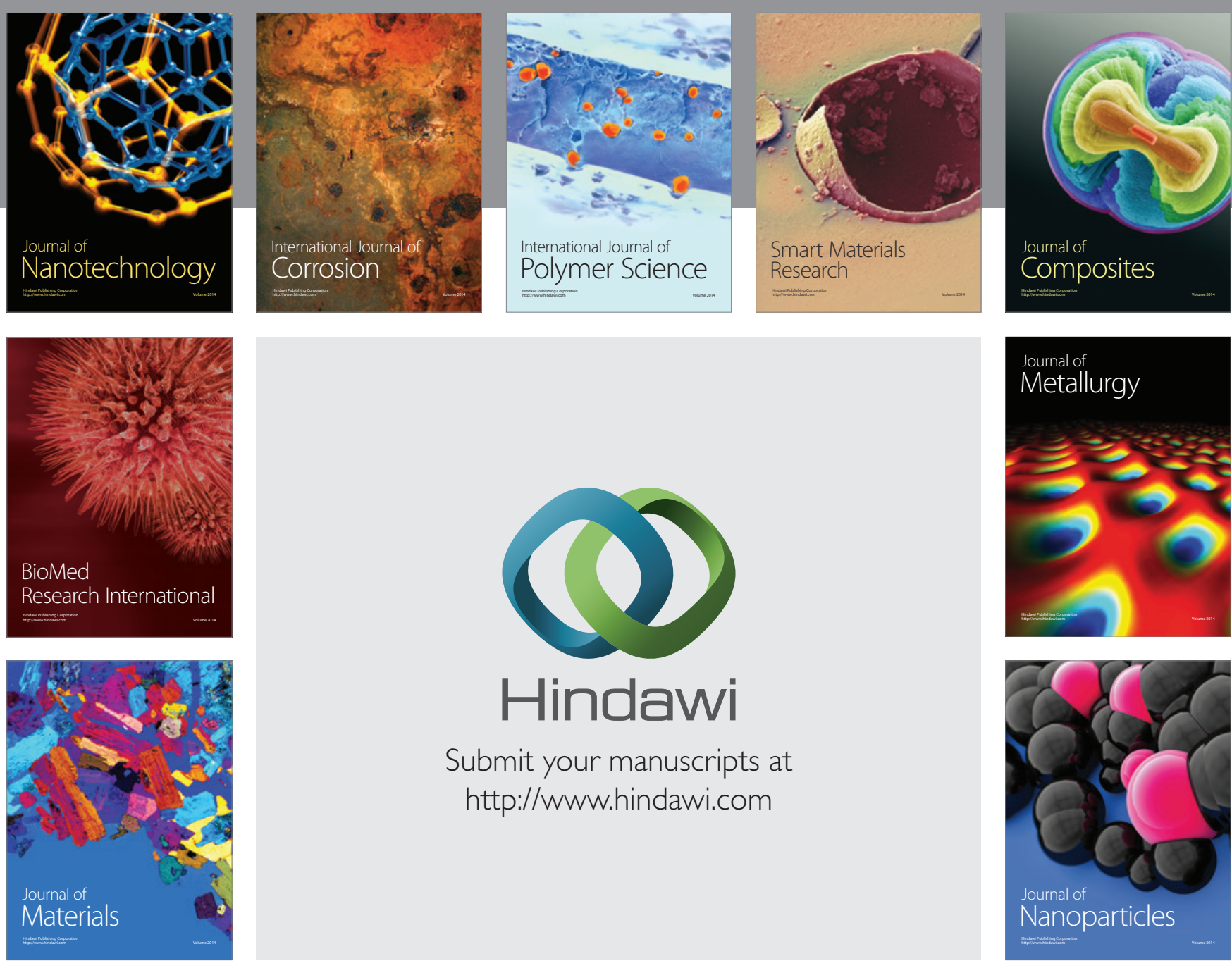

\section{Hindawi}

Submit your manuscripts at

http://www.hindawi.com

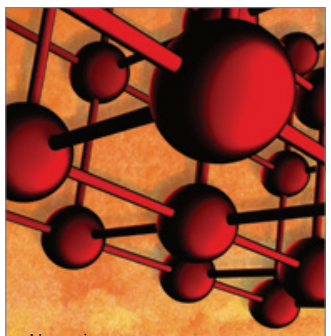

Materials Science and Engineering
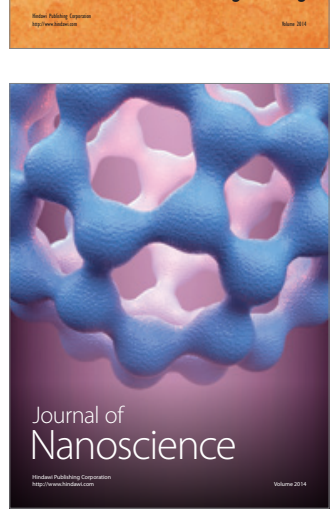
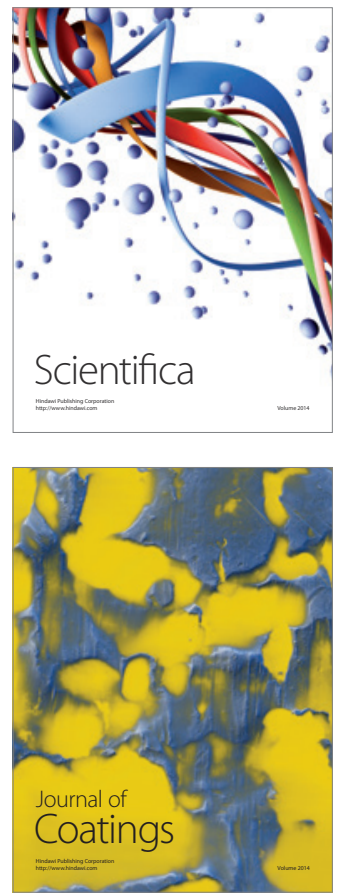
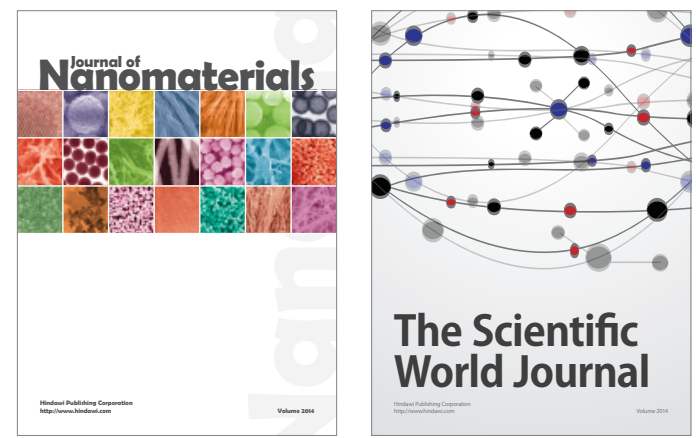

The Scientific World Journal
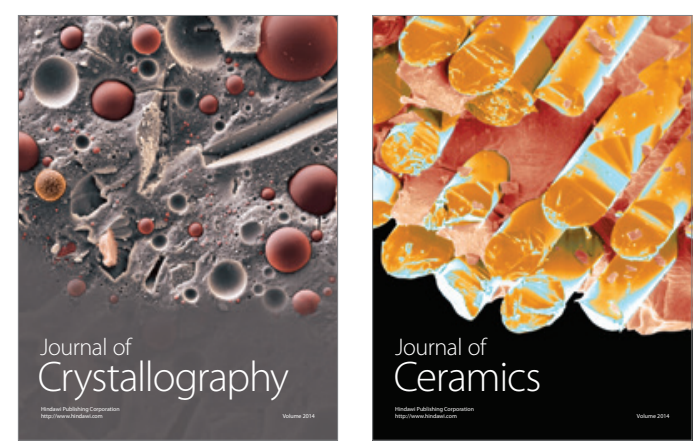
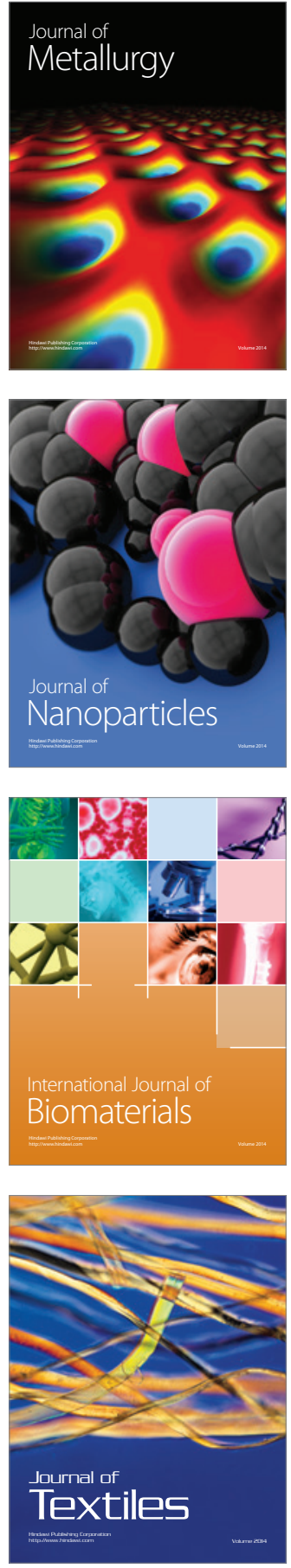